\title{
Model-Based Self-Tuning Multiscale Method for Combustion Control
}

Dzu K. Le, John C. DeLaat, Clarence T. Chang, and Daniel R. Vrnak

Glenn Research Center, Cleveland, Ohio 
Since its founding, NASA has been dedicated to the advancement of aeronautics and space science. The NASA Scientific and Technical Information (STI) Program Office plays a key part in helping NASA maintain this important role.

The NASA STI Program Office is operated by Langley Research Center, the Lead Center for NASA's scientific and technical information. The NASA STI Program Office provides access to the NASA STI Database, the largest collection of aeronautical and space science STI in the world. The Program Office is also NASA's institutional mechanism for disseminating the results of its research and development activities. These results are published by NASA in the NASA STI Report Series, which includes the following report types:

- $\quad$ TECHNICAL PUBLICATION. Reports of completed research or a major significant phase of research that present the results of NASA programs and include extensive data or theoretical analysis. Includes compilations of significant scientific and technical data and information deemed to be of continuing reference value. NASA's counterpart of peerreviewed formal professional papers but has less stringent limitations on manuscript length and extent of graphic presentations.

- TECHNICAL MEMORANDUM. Scientific and technical findings that are preliminary or of specialized interest, e.g., quick release reports, working papers, and bibliographies that contain minimal annotation. Does not contain extensive analysis.

- CONTRACTOR REPORT. Scientific and technical findings by NASA-sponsored contractors and grantees.
- CONFERENCE PUBLICATION. Collected papers from scientific and technical conferences, symposia, seminars, or other meetings sponsored or cosponsored by NASA.

- SPECIAL PUBLICATION. Scientific, technical, or historical information from NASA programs, projects, and missions, often concerned with subjects having substantial public interest.

- TECHNICAL TRANSLATION. Englishlanguage translations of foreign scientific and technical material pertinent to NASA's mission.

Specialized services that complement the STI Program Office's diverse offerings include creating custom thesauri, building customized databases, organizing and publishing research results ... even providing videos.

For more information about the NASA STI Program Office, see the following:

- Access the NASA STI Program Home Page at http://www.sti.nasa.gov

- E-mail your question via the Internet to help@sti.nasa.gov

- Fax your question to the NASA Access Help Desk at 301-621-0134

- Telephone the NASA Access Help Desk at 301-621-0390

- Write to:

NASA Access Help Desk

NASA Center for AeroSpace Information 7121 Standard Drive

Hanover, MD 21076 
NASA/TM-2006-213855

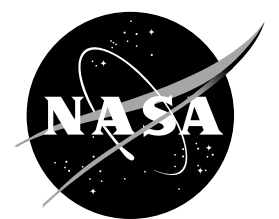

\section{Model-Based Self-Tuning Multiscale Method for Combustion Control}

Dzu K. Le, John C. DeLaat, Clarence T. Chang, and Daniel R. Vrnak

Glenn Research Center, Cleveland, Ohio

Prepared for the

41st Joint Propulsion Conference and Exhibit

cosponsored by the AIAA, ASME, SAE, and ASEE

Tucson, Arizona, July 10-13, 2005

National Aeronautics and

Space Administration

Glenn Research Center 


\section{Acknowledgments}

This research has been funded under the NASA Ultra Efficient Engine Technologies (UEET) project.

Available from

NASA Center for Aerospace Information 7121 Standard Drive

Hanover, MD 21076
National Technical Information Service 5285 Port Royal Road Springfield, VA 22100

Available electronically at http:/ /gltrs.grc.nasa.gov 


\title{
Model-Based Self-Tuning Multiscale Method for Combustion Control
}

\author{
Dzu K. Le, John C. DeLaat, Clarence T. Chang, and Daniel R. Vrnak \\ National Aeronautics and Space Administration \\ Glenn Research Center \\ Cleveland, Ohio 44135
}

\begin{abstract}
A Multi-Scale representation of the combustor dynamics was used to create a self-tuning, scalable controller to suppress multiple instability modes in a liquid-fueled aero engine-derived combustor operating at engine-like conditions. Its self-tuning features designed to handle the uncertainties in the combustor dynamics and time-delays are essential for control performance and robustness. The controller was implemented to modulate a high-frequency fuel valve with feedback from dynamic pressure sensors. This scaleable algorithm suppressed pressure oscillations of different instability modes by as much as 90 percent without the "peak-splitting" effect. The self-tuning logic guided the adjustment of controller parameters and converged quickly toward phase-lock for optimal suppression of the instabilities. The forced-response characteristics of the control model compare well with those of the test rig on both the frequency-domain and the time-domain.
\end{abstract}

\section{Nomenclature}

A $2 \mathrm{~m} \times 2 \mathrm{~m}$ "state-space" matrix in the finite-difference equation of $\mathrm{m}$ acoustics modes (Eq. (1a))

$T \quad$ control loop-time, also taken as time step in the "state-space" equation of the "plant" model

$n \quad$ discrete time index associated with $T$

$u_{n} \quad$ valve-commands for fuel modulation (in Volts)

$b \quad$ expected valve effectiveness on fuel-flow modulation (in 1 /Volt, so that " $b u_{n}$ " is dimensionless)

$i$ (or $j$ ) index of the modeled modes, starting with " $i=1$ " for the fundamental mode

$\omega_{1} \quad$ approximate natural frequency (in radians/second) of combustor acoustics mode $i$

$\zeta_{i} \quad$ approximate damping coefficient for acoustics mode $i$ ("critically damped" is when " $\zeta=1$ ")

$X_{n} \quad 2$ m-vector of m pairs of "quadratures" (dimensionless, modal state-variables) for m acoustics modes

$Q \quad 2$ m-vector function of many variables to model heat-fluctuation lumped effects on acoustics

(Eq. (1a))

$\kappa \quad$ dimensionless gain parameter for the effect of fuel-flow perturbations on heat-release

$\tau \quad$ time-shift operator, also interchangeably denotes the associated (possibly random) delay-time

$v($ or $w)$ wide-band, random disturbances

$p_{o} \quad$ reference pressure differential, in psi, comparable to the pressure drop across the injector

$p_{n}^{\prime} \quad$ combustion zone pressure oscillations (about a mean), in "psi” (pounds per square inch)

$S_{n}^{i} \quad 2$ m-vector of m pairs of "quadratures" extracted from feedback signals $\left\{s_{n}\right\}$ using quadratures filters

$\widetilde{q}_{n} \quad 2$ m-vector of state-variables in the dynamic equation to project $X_{n}$ ahead $N$ time-steps, given $\left\{S_{n}^{i}\right\}$

$\hat{q}_{n} \quad$ a prediction for $X_{n}$ by a Kalman estimate of $\widetilde{q}_{n}$ using $S_{n}^{i}$ as measurement feedback

\section{Introduction}

Lean-burning front-ends (e.g., lean-burn direct injection) for combustors offer the possibility to drastically reduce particulate and $\mathrm{NO}_{\mathrm{x}}$ emissions by aero-engines. The dynamics of this type of combustor, however, are susceptible to instability as has been observed in ground-based lean-burning power generators. This is due to the reduced internal damping and the increased perturbations from more energetic mixing of liquid-fuel and air. The more homogeneous acoustic medium in a typical lean-burn combustor is favorable for well-formed acoustics modes. 
In addition, reduction or elimination of film-cooling liners in the combustor will result in acoustically hard boundary conditions which reflect the perturbations in a coherent manner and further reduce damping. Also, the vigorous mixing of liquid fuel and air could generate energetic perturbations that might overcome the injector relative pressure-drop. Consequently, acoustic pressure oscillations can occur inside the combustor and couple with the fuelair feed-system dynamics to result in non-steady heat-release that, in turn, will amplify the combustion acoustics. Thus, large pressure oscillations could occur inside the combustion chamber, disrupting flame stability and interfering with other subsystems (Ref. 1). Active Combustion Control (ACC) via fuel-modulation provides a method to suppress combustion instabilities to enable the effective operation of such lean-burning combustors for aero-engines (Ref. 2).

The development of the model-based, self-tuning control for fuel-modulation to be presented in this paper is a part of the research on ACC at NASA Glenn to enable direct-injection lean combustion. The interested readers are also referred to other related reports from NASA Glenn (Refs. 3 to 5) for further information on this research, including a more detailed description of the research combustor (named the "NASA SNR"; Figs. 1 and 2), its control system, and other useful findings on combustion control dynamics with this test rig.

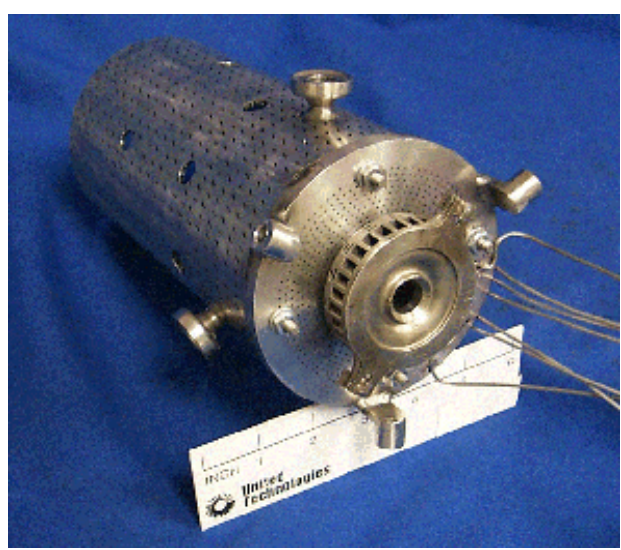

Figure 1.-The single nozzle combustor.

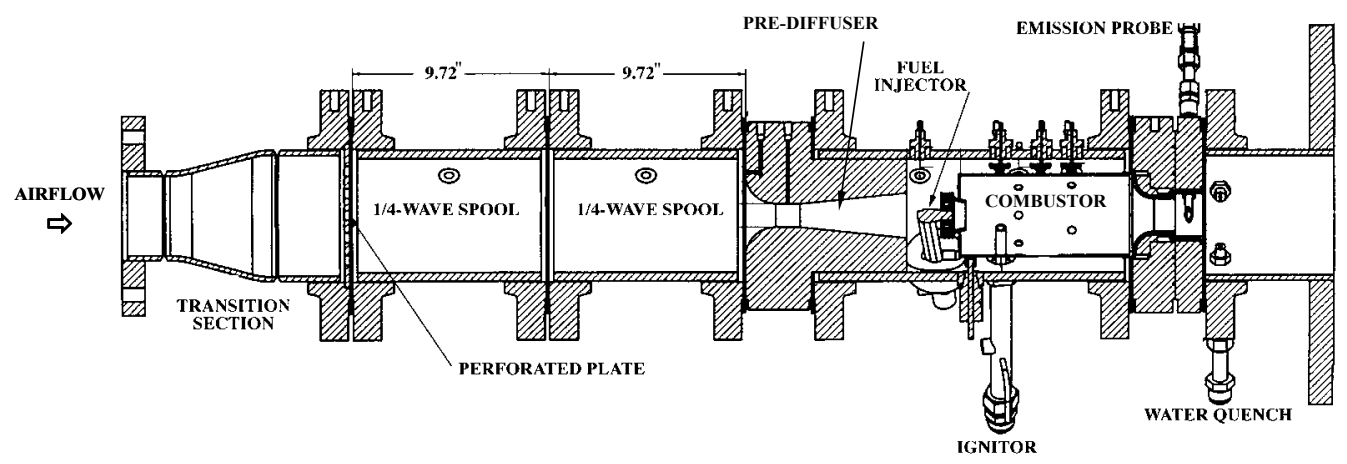

Figure 2.- Schematic of the NASA SNR (in the HF configuration). 


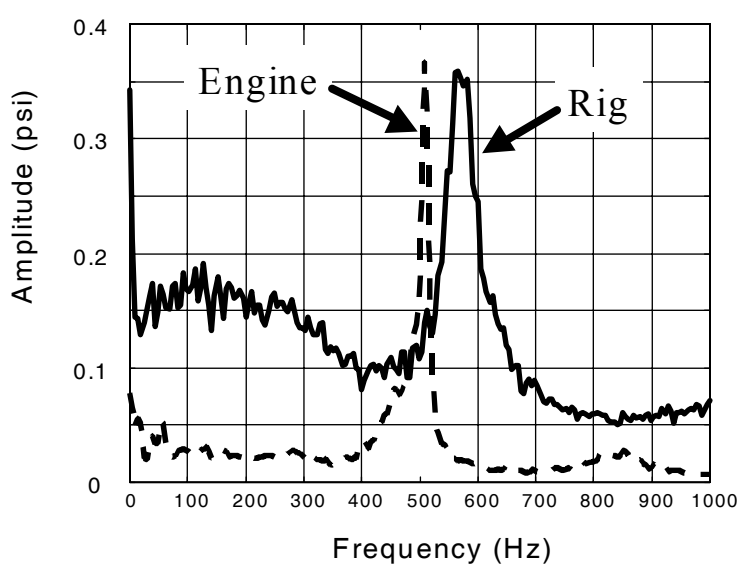

Figure 3.-Engine and NASA HF SNR comparison.

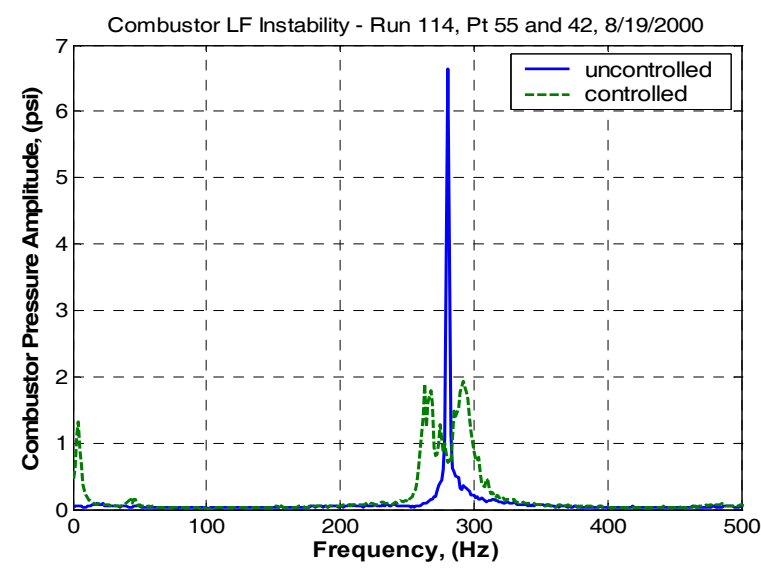

Figure 4.-A case of severe "peak-splitting" under active combustion control for the LF instability (Ref. 13).

In this direction, a model-based controller was developed to operate a high-frequency fuel-modulation valve based on dynamic pressure sensors as feedback to directly affect the instantaneous fuel-air ratio in order to suppress the combustion instabilities. This model-based control method for ACC (named the "Multi-Scale Extended Kalman" approach, abbreviated MSEK (Ref. 6)) uses multi-scale analyses (Refs. 7 and 8) and an extended Kalman filter (Refs. 9 and 10) to predict and control fuel-modulation effects and the combustion thermo-acoustics. A prototype of the MSEK core logic (named "the Basic MSEK" throughout this paper) was demonstrated in 2002 on a test rig for an instability with high-frequency pressure oscillations (greater than $500 \mathrm{~Hz}$ ) (Ref. 6). This NASA Glenn single nozzle combustion rig operates at engine pressures, temperatures, and flows using liquid jet fuel and incorporates many engine-like features, including and actual engine fuel nozzle and swirls, dilution cooling, and an effusioncooled liner (Ref. 11). The rig (Figs. 1 and 2) was developed in partnership with Pratt \& Whitney and United Technologies Research Center. Although the rig exhibited a higher level of background noise/disturbances than observed in the engine (Fig. 3), it adequately replicated an observed engine instability experience. In addition to the baseline configuration at "high-frequency" (HF) the combustor rig can also be changed to a "low-frequency" (LF) and "mid-frequency" (MF) configuration. For the LF configuration, which exhibits $\sim 280 \mathrm{~Hz}$ instability, both spool pieces, as shown in Figure 2 are moved to a position between the pre-diffuser and fuel injector. For the MF configuration, which exhibits a $\sim 340 \mathrm{~Hz}$ instability, only one spool piece is moved to between the pre-diffuser and fuel injector. The initial investigation of the Basic MSEK with the NASA SNR in the HF configuration was conducted in May and September 2002. This initial investigation, performed with both the Basic MSEK and an adaptive controller by Kopasakis (Ref. 5), constituted the first successful demonstration of ACC on a realistic aeroengine combustion rig (Refs. 5, 6, and 12). Prior to that demonstration, partial success in suppressing the LF instability had been reported (Ref. 13). The main issue with control-performance in this and other earlier works was the so-called "peak-splitting" phenomenon (as illustrated by the pressure amplitude-spectra of Fig. 4). While the dominant instability was suppressed, other instability peaks arose around the central frequency; and consequently, the reduction in oscillation rms amplitude was poor.

Such problems (perhaps due to both the valve dynamics and particular control strategies) can be overcome with a high-performance valve and by using variable control gains and other real-time adjustment of control parameters, as recently demonstrated with the "Self-Tuning MSEK". In this follow-on development of the MSEK, various parameter optimizations and "self-tuning triggers" were used to effectively minimize the time-scale averaged variances of the combustion sensed pressure without aggravating the existing instability at any time. Such selftuning features (based on multi-scale variances or higher moments of pressure oscillations for the triggers to adjust the parameters and setting their increments and search ranges) are essential for handling the severe uncertainties inherent in combustion time-delays and other characteristics, while keeping the embedded predictor model in the controller simple. The resulting Self-Tuning MSEK controller showed good results on the $M F$ and $L F$ configurations of the NASA SNR, including a very fast convergence towards optimal "phase-lock." For the LF SNR configuration, where the signal-to-noise ratio is high, the Self-Tuning MSEK controller was able to reduce the amplitude-spectrum peak by 90 percent as well as the overall pressure oscillations rms value by 80 percent without causing peaksplitting.

This paper describes the improvements recently made on the Basic MSEK to create the Self-Tuning MSEK. In sections II and III, the reduced-order model and validation results based on some open-loop forced-response data of the SNR are presented. The MSEK controller formulation, self-tuning features, and ACC demonstration results on 
the SNR are presented in sections $I V$ and $V$. Some conclusions and highlights of these ACC experiments, and control and model developments are given in section VI.

\section{Reduced-Order Thermo-Acoustics Model}

The reduced-order thermo-acoustics model for this control development, the same as presented in the previous report for the 2002 work (Ref. 6), is reviewed here for further clarification. Except for parameter values, this same model is applicable for all three configurations (HF, MF, and LF) of the SNR. It is formulated for the two dominant thermo-acoustics modes of the SNR as follows (see nomenclature list for the variables description):

$$
\begin{gathered}
X_{n+1}=A X_{n}+Q\left(\tau_{a} X_{n,} \tau_{b} \widetilde{u}_{n,} \tau_{c} P_{n,} W_{n}\right) ; \\
p_{n}^{\prime}=\left[\begin{array}{llll}
c_{1} & 0 & c_{2} & 0
\end{array}\right] X_{n} \\
S_{n}=p_{n}^{\prime}+V_{n}
\end{gathered}
$$

In equation (1a), the $4 \times 4$ state matrix $A$ is approximated by the following expression, with $\lambda_{\mathrm{i}} \equiv \exp \left(-2 \zeta_{\mathrm{i}} \omega_{\mathrm{i}} T\right)$ :

$$
A=\left[\begin{array}{cc}
\lambda_{1}\left[\begin{array}{cr}
\cos \left(\omega_{1} T\right) & -\sin \left(\omega_{1} T\right) \\
\sin \left(\omega_{1} T\right) & \cos \left(\omega_{1} T\right)
\end{array}\right] & {\left[\begin{array}{c}
0 \\
{[}
\end{array}\right]} \\
{\left[\begin{array}{ll}
0 & ]
\end{array}\right.} & \lambda_{2}\left[\begin{array}{rr}
\cos \left(\omega_{2} T\right) & -\sin \left(\omega_{2} T\right) \\
\sin \left(\omega_{2} T\right) & \cos \left(\omega_{2} T\right)
\end{array}\right]
\end{array}\right.
$$

Typically, $\omega_{1}$ ("Mode 1 ") could be $2 \pi \times 290$ radians/sec to $2 \pi \times 340$ radians $/ \mathrm{sec}$, depending on the rig; and $\omega_{2}$ ("Mode 2") could be $2 \pi \times 520$ radians/sec or higher. The two damping coefficients $\zeta_{\mathrm{i}}$ (for $\left.\mathrm{i}=1,2\right)$ are typically about 0.01 to 0.05 . The heat-release fluctuation, $Q$, affecting the acoustics is a $4 \times 1$ vector-valued function of delayed acoustics states, internal pressure feedback on fuel-flow, and fuel-modulating valve position $\tilde{u}_{n}$ (about a mean position); with delay effects modeled by random time-shift operators $\tau_{\mathrm{a}}, \tau_{\mathrm{b}}, \tau_{\mathrm{c}}$ (of non-zero mean values) applied on the various variables. The calculation of $Q$ with these variables is described in the following intermediate steps, in which the parameters $\left\{h_{\mathrm{i}}\right\}$ and " $g$ " are constant, internal feedback gains; " $b$ " represents the effectiveness of fuelmodulating on pressure oscillations; " $p_{\mathrm{o}}$ " a reference pressure comparable to the mean pressure drop across the injector:

$$
\left[\begin{array}{c}
p_{1} \\
p_{2}
\end{array}\right] \equiv\left[\begin{array}{llll}
h_{11} & 0 & h_{12} & 0 \\
h_{21} & 0 & h_{22} & 0
\end{array}\right] \tau_{a}\left(X_{n}\right)+\left[\begin{array}{lll}
b & g & 1 \\
b & g & 1
\end{array}\right]\left[\begin{array}{c}
\tau_{b}\left(\tilde{u}_{n}\right) \\
\tau_{c}\left(p_{n}\right) \\
w_{n}
\end{array}\right]
$$

subject to bounds : $p_{\min }^{\prime} \leq p_{1}, p_{2} \leq p_{\max }^{\prime}$

$$
\begin{gathered}
Q_{i}=\kappa_{i}\left(\sqrt{\left(p_{i} / p_{o}\right)+1}-1\right)(\text { for } i=1,2) \\
Q \equiv\left[\begin{array}{llll}
0 & Q_{1} & 0 & Q_{2}
\end{array}\right]^{t}
\end{gathered}
$$

The superscripted operator $[\ldots]^{t}$ as in Equation (1g) and elsewhere denotes matrix/vector transpose. The imposed bounds on $p_{1}$ and $p_{2}$ following Equation (1e), for the calculation of $Q$, was implemented differently by substituting the following expressions instead of $Q_{\mathrm{i}}$ (for $\left.i=1,2\right)$ into $(1 \mathrm{~g})$ :

$$
\left(2 U_{i} / \pi\right) \arctan \left(0.5 \pi\left(Q_{i}+e\right) / U_{i}\right)
$$


The inputs $Q_{i}$ for expression (1h) are defined as in (1f), except that the bounds previously imposed on $p_{\mathrm{i}}$ are no longer needed (Instead, the quantity $0 \leq U_{\mathrm{i}} \leq 1$, for $\mathrm{i}=1,2$, are constant fractions now provide the necessary upperbounds for the quantity (1h)); and, "e" is a bias. These parameters as well as $\left\{h_{\mathrm{i} j}\right\}, b$, and $\kappa$ will be chosen to match the open-loop forced response characteristics of the combustion chamber pressure (see section III). Note that, the dynamics of the heat-fluctuation term $Q$ is very complex, as it is the result of heat convection, diffusion, air flow and fuel flow, and combustion. The simplistic approximation of $Q$ in (1e) to (f), with some imposed bounds (empirical, implemented as in (1h)), and the Euler expression for the fuel-air ratio fluctuations as in (1f) is only for modeling its average (non-linear) effects on the acoustics. For example, the model data for the Low-Frequency Configuration as set-up at Glenn in 2004 are chosen as follows:

First, choose $p_{\mathrm{o}}=20.0 \mathrm{psi} ; T=0.0002 \mathrm{sec} ; \kappa_{1}=1.2 p_{\mathrm{o}}$ $=24.0 ; \kappa_{2}=0.4 p_{\mathrm{o}}=8 ; g=0.001 ;$ and, $b=0.3$. Next, $\omega_{1}=1822 \mathrm{radians} / \mathrm{sec} ; \zeta_{1}=0.042 ; c_{1}=1.0 ; h_{11}=1.0$; $U_{1}=0.9$; and, $e_{1}=2.64$ (Mainly for matching mode-1 characteristics). Then, $\omega_{2}=3456$ radians $/ \mathrm{sec} ; \zeta_{2}=0.01$; $c_{2}=1.0 ; h_{22}=1.0 ; U_{2}=0.3 ; e_{2}=3.2$ (For mode- 2 characteristics). And lastly, $h_{1} 2_{2}=0.1 ; h_{2}{ }_{1}=2.0$ (The influence of mode 1 on mode 2 appeared strong in the LF SNR); $\bar{\tau}_{\mathrm{a}}=0.0038 ; \quad \bar{\tau}_{\mathrm{b}}=0.0033$; $\bar{\tau}_{\mathrm{c}}=0.0002 \mathrm{sec}$ (Empirical, mean values).

Model validation with this parameter set will be presented in the next section.

\section{Model Validation With the "SNR" Test Data}

The reduced-order model and associated parameters for the HF configuration of the SNR had been validated in an earlier work (Ref. 6). Therefore, this section will be devoted only to validation of model for the $L F$ configuration (LF) of the SNR. This configuration is particularly important for validating the model, because its internal instability is much more prominent compared to the external disturbances. The latest MSEK and self-tuning features were built and enhanced mainly with modeling data from this test rig configuration. The characteristics of the LF SNR self-sustained instabilities and open-loop responses to fuel-modulation by a high-frequency valve (Fig. 5) are shown next to validate the described reduced-order plant model and its parameter values. To highlight the agreement of model results with test data, this validation will be shown here with more refined parameters than typically done for preliminary control tuning prior to testingpreliminary tuning of control parameters prior to testing on the rig required only rough estimates of model parameters on the instability magnitude and the valve "control-authority" (i.e., the response characteristics of the combustor pressure under open-loop commands).

Some typical non-linear response characteristics of the LF SNR under sinusoidal fuel-modulation with the high-frequency fuel-valve are shown in Figures 6 and 7. In these figures, the signal named
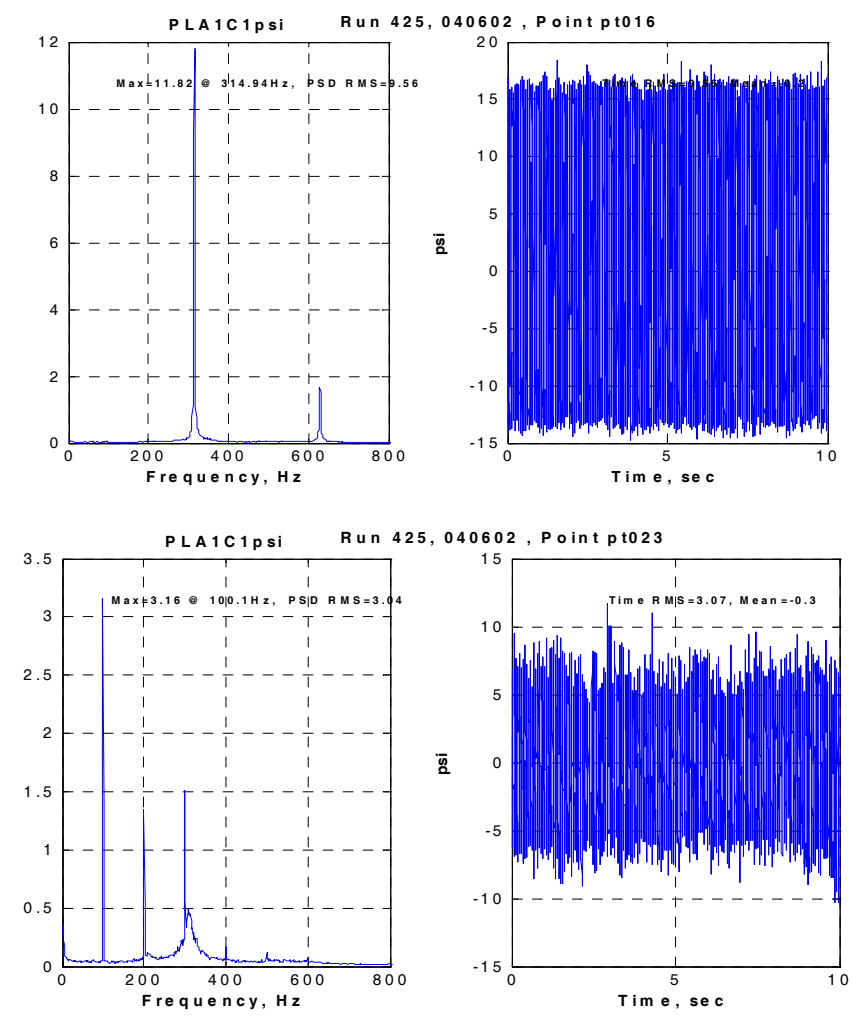

Figure 6.-(Upper subplots) The LF SNR self-sustained instabilities (oscillations in psi); (Lower) Effects of $100 \mathrm{~Hz}$ sinusoidal valve commands with $3-\mathrm{V}$ amplitude on the instabilities, resulting in about $10 \mathrm{psi}$ change in pressure oscillations. 

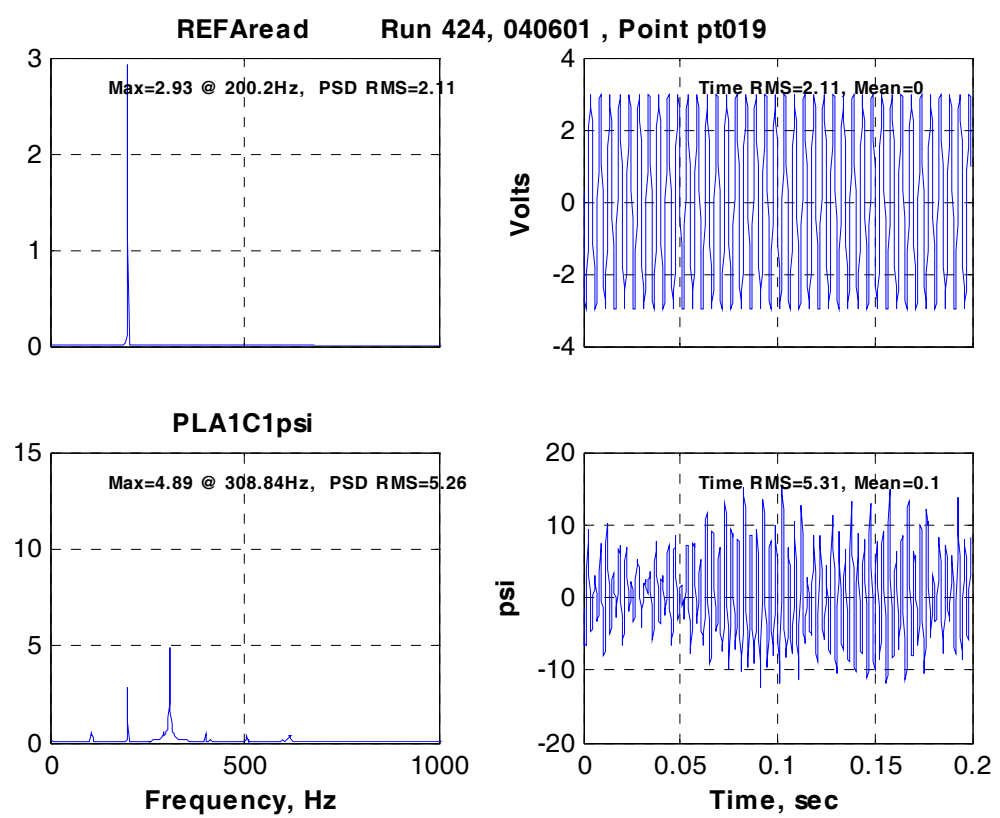

Figure 7.-Effects of $200 \mathrm{~Hz}$ sinusoidal valve commands of 3-V amplitude on the LF SNR instabilities, resulting in about 7 psi change in pressure oscillations.
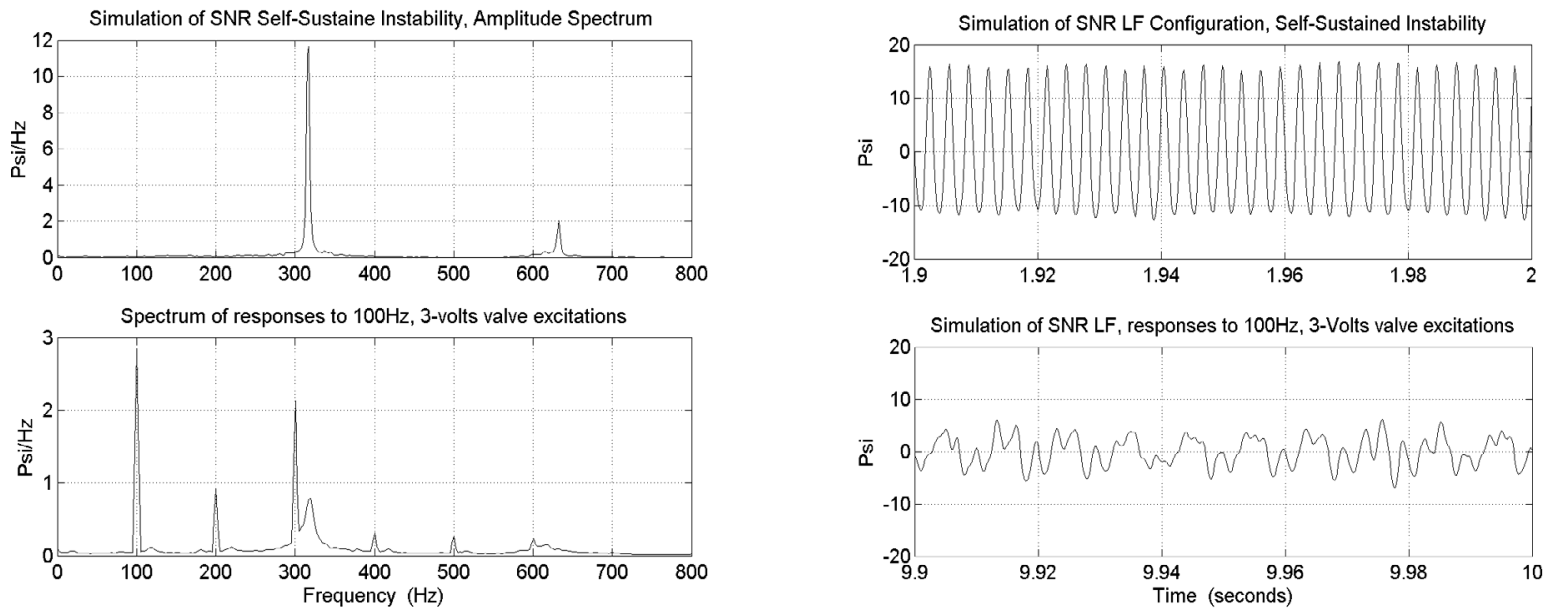

Figure 8.-(Upper subplots) Model results of LF self-sustained instabilities; (Lower) Effects of $100 \mathrm{~Hz}$ sinusoidal valve commands of 3-V amplitude on the LF SNR Model instabilities.

"PLA1C1" is the combustion pressure oscillation signals sensed at about 2 inches downstream of the fuel injector; and "REFAread" (in Volts) represents the valve command. For example, the two upper subplots of Figure 6 show the amplitude spectra and time-domain trace of the combustor pressure self-sustained oscillations (in psi) without fuel-modulation. The main instability frequency is about $315 \mathrm{~Hz}$. The higher frequency mode, at about $620 \mathrm{~Hz}$, is also visible on the amplitude spectra plot. The two lower subplots of Figure 6 show the responses of the internal pressures to $100 \mathrm{~Hz}, 3-\mathrm{V}$ sinusoidal open-loop commands on the valve. The lower subplots in Figure 6 as well as Figure 7 (for $200 \mathrm{~Hz}$ open-loop inputs) clearly show the nonlinear response characteristics of the combustion instabilities and the significant effects of the fuel-valve pulsation. 
Figure 8 shows the simulation data of the LF SNR reduced-order model to be compared to the test data of Figure 6. It shows that, the modeled self-sustained instabilities as well as their responses to the $100 \mathrm{~Hz}, 3-\mathrm{V}$ sinusoidal fuel-modulation open-loop commands, including non-linear characteristics, compare well on both the frequency-domain and the time-domain with the test data of the rig. Likewise, the model results of Figure 9 compare well with the test results shown in Figure 7.

\section{The Self-Tuning MSEK Controller}

The top-level structure of the Self-Tuning MSEK controller for ACC is still the same as that of the "basic MSEK"(Ref. 6) tested in 2002 on the HF SNR (see Fig. 10). The control scheme formulation, and additional self-tuning features as in the latest version will be described in more detail in the following sub-sections. The
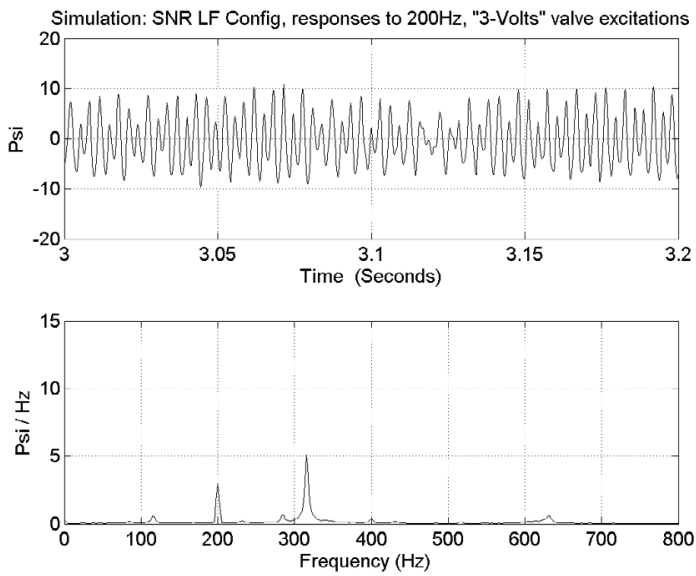

Figure 9.- -Response characteristics of the LF SNR Model to $200 \mathrm{~Hz}$ valve commands of 3-V amplitude. subsection titles refer to the individual sub-block names in Figure 10. Except for the last subsection (Automatic Tuning of Critical Control Parameters), these subsections are reviews of the basic control scheme that had been briefly described in the previous work (Ref. 6).

\section{A. Multi-Scale Tone Analysis Using "QA"}

This pre-filtering part of controller MSEK (Fig. 10) is performed by a "Quadratures Filter Bank" of wavelet-like filters to extract the dominant "tones" of the sensed pressures. By definition, a "Quadrature Analysis" (QA) on a frequency range of a signal stream $\left\{\mathbf{S}_{n}\right\}$ (a time-series with $n$ being the time index) is any decomposition of the signal contents within that range into two mutually orthogonal components $\left\{S_{1 n}\right\}$ and $\left\{S_{2 n}\right\}$ by using an Orthogonal Wavelets Decomposition and Partial Reconstruction scheme (e.g., using "quadrature mirror filters") (Ref. 7). For simplicity, instead of such a wavelet scheme, a pair of Finite Impulse Response (FIR) band-pass filters is designed for each frequency range to extract (approximate) "quadratures" from the signal: One filter with unity gain and 45-degrees lead at the center of the frequency range of interest, to extract the time-series $\left\{S_{1 n}\right\}$; and another, also with unity gain, but with 45-degrees lag at the same frequency, to extract $\left\{S_{2 n}\right\}$. Using the described QA process, for each of the two main frequency ranges (LF and HF) of the SNR, a "filter-bank" consist of three Quadrature Filter pairs (hence a minimum of six filters for each range) was used to analyze the sensed pressure oscillations and, for each time point $n$, extract the dominant pair among the three pairs of quadrature components. In particular, for the LF range, one pair of Quadrature Filter as described is designed for the central LF, and the other two are for $0.98 \mathrm{LF}$ and 1.02 LF, respectively. These are then used to extract the dominant LF QA sequence, denoted $\left\{S_{n}^{1} \equiv\left[S_{1 n}^{1} S_{2 n}^{1}\right]^{t}\right\}$ as in the formulation of the predictor dynamics in the next section. Likewise is done to extract the HF QA sequence which will be denoted $\left\{S_{n}^{2} \equiv\left[S_{1 n}^{2} S_{2 n}^{2}\right]^{t}\right\}$. The advantage of this (approximate) QA process is its simplicity; also, the polar angle of the vector [ $\left.\begin{array}{ll}S_{1 n}^{\mathrm{i}} & S_{2 n}^{\mathrm{i}}\end{array}\right]^{t}$ (being the phase angle of $\left\{S_{1 n}^{\mathrm{i}}\right\}$ ) represents the instantaneous "phase" angle of the tone $i$ plus $\pi / 4$ radians.

\section{B. EK States Prediction}

Although the evolution equation for $S_{n}^{\mathrm{i}}$ can be derived from the state-equations (1a) to (1h) to develop the prediction scheme, such route is avoided because it is excessively complicated for real-time calculation. Thus, the state-equations (1a) to (1h) will be used directly in the following development of the Predictor (Eqs. (3a) to (3e)). Note also that, if the "QA" process is applied to the output $\left\{s_{n}\right\}$ simulated by the model in equation (1c), then the resulting $S_{n}^{i}$ is approximately $c_{i} X_{n}^{i}$ (for $i=1,2$ ) with about 45-degree phase-lead. Hence, the following fictitious random process would be a good projection of the actual state variables $X_{n}$ ahead $N$ sampling steps (such that, $N \times T$ $\sim$ total delay-time) if certain augmented input " $L$ " can be designed based on $S_{n}^{\mathrm{i}}$ (for $i=1,2$ ) to make the output $y_{n}$ track the measurable output " $c_{1} X_{n}^{1}+c_{2} X_{n}^{2}$ " despite the random disturbances $w$ and $v$ : 


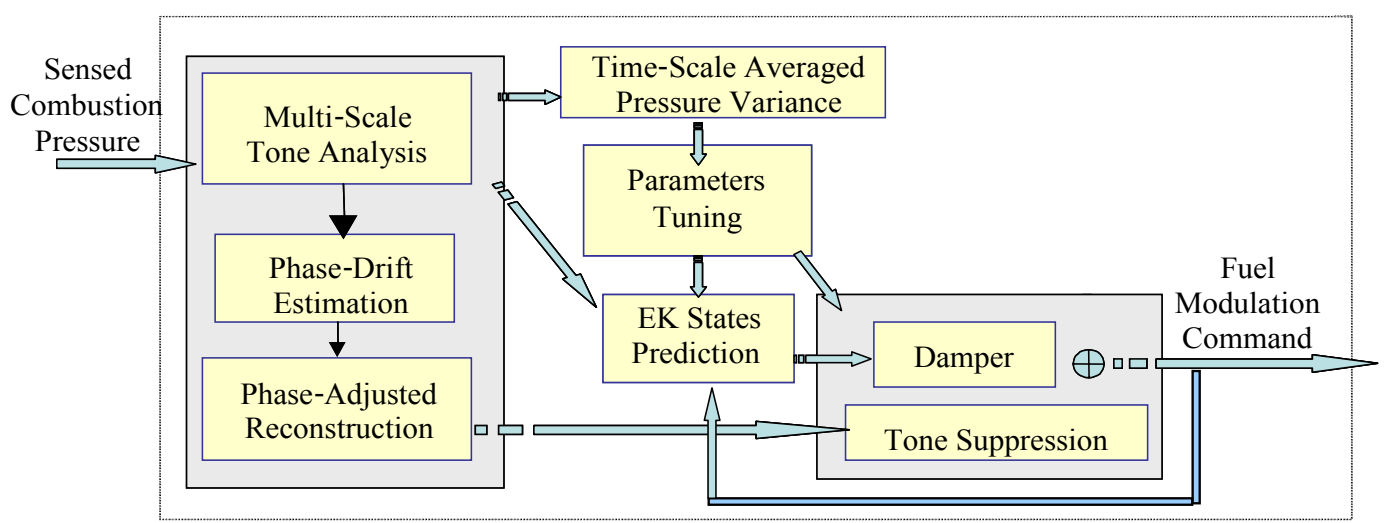

Figure 10.- The MSEK controller functional block diagram.

$$
\begin{gathered}
\widetilde{q}_{n+1}=A \widetilde{q}_{n}+\widetilde{Q}+w+L\left(S_{n}^{1}, S_{n}^{2}\right) \\
y_{n} \equiv c_{1} \widetilde{q}_{n-N}^{1}+c_{2} \widetilde{q}_{n-N}^{2}+v
\end{gathered}
$$

The random effects $w$ and $v$ need to be included here in the design of the Kalman filter for $\widetilde{q}_{n}$ estimation, so that the predictions would be robust against disturbances. Also, to simplify the Kalman filter, the projected " $Q$ "-term shown in Equation (2a) will be approximated using $S_{n}^{1}$ (for $i=1,2$ ) (but, without time-delays in the formulation; (see Eqs. (3c) and (3d)) rather than by integration as part of the above process. Furthermore, the first two terms of the right-hand-side of Equation (2b) add up to $M \widetilde{q}_{n_{-N}}$, where $\mathrm{M} \equiv\left[c_{1} I, c_{2} I\right]$, and " $P$ " denotes the $2 \times 2$ identity matrix. So, if the 5th-order Pade approximation is used for the N-step delay to apply on the 2-vector $M \widetilde{q}_{n}$ to compute the delayed sequence, the combined state matrices of that transformation individually applied on the two vector components would result in the state-space matrices $U, F, E, D$ (of dimensions $10 \times 10,10 \times 2,2 \times 10$, and $2 \times 2$, respectively) that yield the following reformulation of Eq. (2b):

$$
\begin{gathered}
z_{n+1}=U z_{n}+F M \widetilde{q}_{n} \\
y_{n} \approx E z_{n}+D M \widetilde{q}_{n}+v
\end{gathered}
$$

Equations (2a), (2c), and (2d) result in the following Quadratures Kalman Predictor obtained by estimating $\widetilde{q}_{n}$; the estimation is denoted $\hat{q}_{n}$ for simplicity, and meant as a projection for $X_{n}, N$ time-steps ahead:

$$
\begin{aligned}
\hat{q}_{n+1} & =A \hat{q}_{n}+\hat{Q}+K_{1}\left(E \hat{Z}_{n}+D M \hat{q}_{n}-S_{n}^{1}-S_{n}^{2}\right) \\
\hat{Z}_{n+1}= & U \hat{Z}_{n}+F M \hat{q}_{n}+K_{2}\left(E \hat{Z}_{n}+D M \hat{q}_{n}-S_{n}^{1}-S_{n}^{2}\right) \\
& {\left[\begin{array}{l}
\hat{p}_{1} \\
\hat{p}_{2}
\end{array}\right] \equiv\left[\begin{array}{llll}
k_{11} & 0 & k_{12} & 0 \\
k_{21} & 0 & k_{22} & 0
\end{array}\right]\left[\begin{array}{l}
S_{n}^{1} \\
S_{n}^{2}
\end{array}\right]+\left[\begin{array}{l}
b \\
b
\end{array}\right] u_{n} } \\
& \text { subjected to }: p_{\text {min }}^{\prime} \leq \hat{p}_{1}, \hat{p}_{2} \leq p_{\text {max }}^{\prime}
\end{aligned}
$$




$$
\begin{gathered}
\hat{Q}_{i} \equiv \kappa_{i}\left(\sqrt{\left(\hat{p}_{i} / p_{o}\right)+1}-1\right) \\
\hat{Q} \equiv\left[\begin{array}{llll}
0 & \hat{Q}_{1} & 0 & \hat{Q}_{2}
\end{array}\right]^{t}
\end{gathered}
$$

In Equation (3c) the parameters " $k_{\mathrm{i} \mathrm{j}}$ " are related to " $h_{\mathrm{ij}}$ " such that: $h_{\mathrm{ij}} \approx c_{i} k_{\mathrm{i}}$ (for $\left.i, j=1,2\right)$, where " $c_{i}$ " and

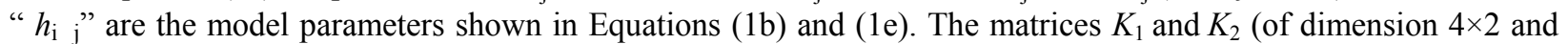
$10 \times 2$, respectively) are the two vertical sub-blocks of the Kalman matrix computed for states estimation, assuming certain covariance matrices for the random vectors $v$ and $w$. Note that, the time-delay of the " $Q$ "-term in the actual process $X_{n}$ was dropped to affect the desired lead time for this prediction scheme (i.e., comparing Eq. (3c) to (1e)). It is also sufficient to use the simple bounds after Equation (3c) instead of the more refined expressions (1h).

\section{The Damper}

If $S \equiv\left\{\left[S_{1 n}, S_{2 n}\right]^{t}\right\}$ denotes the time-series of "quadrature pairs" extracted from a signals $\left\{\mathrm{s}_{n}\right\}$ by a "QA" process as described previously, then the linear combination (associated with an angle $\alpha$, in radian) denoted $S_{\alpha} \equiv\left\{S_{1 n} \cos \alpha-S_{2 n} \sin \alpha\right\}$ represents the extracted tone of $\left\{\mathrm{s}_{n}\right\}$ phase-shifted by " $\alpha+\pi / 4$ " radians. Such combination is used on the predicted quadratures, namely $\widehat{S}_{n}^{i} \equiv c_{i} \hat{q}_{n}^{i}$ (for $i=1,2$ ), to prescribe a Linear Quadratic Regulator-type damper (This part of the fuel-modulation command, as in Fig. 10, is distinguished here by the superscript " $d$," with the time-index " $n$ " suppressed and the superscript being the mode index):

$$
u^{\prime d '}=\mu\left[l \hat{S}_{\beta_{1}}^{1}+(1-l) \hat{S}_{\beta_{2}}^{2}\right]
$$

where "l" is a chosen "mode-1 fraction" (with $0 \leq l \leq 1$ ) (basically a Loop-Shaping means for performance optimization), and " $\mu$ " is the overall gain for the "Damper". The terms $\hat{S}_{\beta_{i}}^{i}($ for $i=1,2)$ are the phase shifted signals

constructed from the predicted "quadratures," using the respective phase angles $\beta_{\mathrm{i}} \equiv \bar{\beta}+\delta_{\mathrm{in}}$ (for $i=1,2$ ), with $\bar{\beta}$ being a certain bias and $\delta_{\mathrm{in}}$, a predicted phase-drift. The drifts $\delta_{\mathrm{in}}$ (over the time interval of expected control transport-delay, for tones $i=1,2$ ) are predicted by a dynamic, linear regression scheme applied on the quadrature phases $\left\{\alpha_{\mathrm{in}}\right\}$ as defined in equation (5) of the next sub-section.

\section{The "Phase-Adjusted Reconstruction" and Tone Suppression}

This part of the control scheme is for countering the non-steady heat-release effects. For this purpose, the instantaneous amplitudes and phases of the combustion pressure near the LF and HF are first estimated by projecting the quadratures pair $S_{n}^{i}$ (for $i=1,2$, as $2 \times 1$ vectors) onto an orthogonal frame rotating at the respective speed $\omega_{1}$ (referring to the frequencies in Equation (1d)). To minimize the effect of random noises, these projections are next passed through low-pass filters with cut-off frequencies at 100 and $200 \mathrm{~Hz}$, respectively, before computing the amplitudes and phases. Note that, these cut-off frequencies are about $1 / 3$ of the respective central frequencies (So that variations in amplitude for every three oscillation at such frequency should be detected). Those estimations form the following expression for feedback to the tone-suppression logic (for modes $i=1,2$ ):

$$
\sigma_{i}=r_{i n} \cos \left(n \omega_{i} T+\alpha_{i n}\right)
$$

where $\left\{r_{\mathrm{in}}\right\}$ is the computed amplitude, and $\left\{\alpha_{\mathrm{in}}\right\}$ the phase angle at time $t_{n}$ of the filtered, projected quadratures vector. Then, $\left\{\alpha_{\mathrm{in}}\right\}$ is used to predict the phase drifts $\delta_{\mathrm{in}}$ (for the expected control transport-delay, for $i=1,2$ ) using a dynamic linear regression scheme. Since the quantities $\sigma_{\mathrm{i}}$ should be highly coherent with the non-steady 
heat-release, the fuel-modulation command $u_{n}$ will include a compensation term (distinguished by the superscript ' $c$ ') of the following form for Tone Suppression (see Fig. 10) to minimize these effects:

$$
u_{n}^{c^{\prime}}=\gamma\left[l \chi_{1 n} r_{1 n} \cos \left(n \omega_{1} T+\alpha_{1 n}+\bar{\alpha}+\delta_{1 n}\right)+(1-l) \chi_{2 n} r_{2 n} \cos \left(n \omega_{2} T+\alpha_{2 n}+\bar{\alpha}+\delta_{2 n}\right)\right],
$$

where " $\gamma$ " is the overall gain for Tones-Suppression and " $0 \leq l \leq 1$ ", the same "mode- 1 fraction" used in Equation (4). A varying factor, $\left\{\chi_{\mathrm{in}}\right\}$ (for $i=1,2$ ), is also introduced to allow for gradual reduction of the ToneSuppression actions when " $r_{\mathrm{i}}$ " is sufficiently small (This is essential for rendering absolute stability to the controlled model as shown in the 2002 study) (Ref. 6). It is basically a unit-gain with an integral adjustment, calculated by digitizing the following expression (also, " $\chi_{\mathrm{in}}$ " is subjected to certain bounds $\chi_{\min }$ and $\chi_{\max }$ to maintain numerical stability):

$$
\chi_{i} \equiv 1+\eta \int\left(r_{i}-\rho_{i}\right) d t
$$

where " $\eta$ " is a constant, and " $\rho_{\mathrm{i}}$ " is a reduction level expected to be maintainable for " $r_{\mathrm{i}}$ " with this scheme. In summary, the Tones-Suppression command is generated with these gains on " $\sigma_{\mathrm{i}}$ " after having phase-shifted it by a bias $\bar{\alpha}$ plus $\delta_{\mathrm{in}}$.

\section{E. Automatic Tuning of Critical Control Parameters}

The fuel-modulation command $\left\{u_{\mathrm{n}}\right\}$ issued to the valve is the sum of the expressions (4) and (6). For all SNR configurations, the parameters in the predictor as well as in the described command scheme appear to be fairly deterministic, except for the phase biases ( $\bar{\alpha}$ and $\bar{\beta}$ ) and the control authority represented by " $b$ " (see Eq. (3c)). The critical control parameters of the MSEK that require automatic tuning are: $\bar{\alpha}, \bar{\beta}, b, \mu$, and $\gamma$. The remaining parameters, including " $l$ " (also of importance) can be preset based on the model, or calibrated empirically (In particular, the parameter " $l$ " can also be searched in real-time automatically, at a very safe and slow pace as demonstrated on the model as in the last subsection of section V (Control Evaluation).

The three parameters $\bar{\alpha}, \bar{\beta}$, and $b$ are determined by the Parameters Tuning logic (Fig. 10) via sequential, globaltype searches. These searches are to minimize the Time-Scale Averaged Pressure Variance (in Fig. 10, obtained by applying a $0.2-\mathrm{Hz}$ low-pass filter on the total square-norm of the two vectors $S^{\mathrm{i}}{ }_{n}$, for $i=1,2$ ). The searches are activated only if performance degrades below a certain threshold (a scalable threshold based on the model). The search neighborhood is also narrowed down if performance is improving. In parallel, fast management of $\mu$, and $\gamma$ (with a common variable factor) are done to avoid aggravating the instabilities at wrong values of $\bar{\alpha}, \bar{\beta}$, and $b$. That is, these gains are dropped quickly to zero when an overshoot trend is detected in the pressure variances near the instability frequencies.

The "Basic MSEK" (the core of the "Self-Tuning MSEK," namely the control actions formulated in Equations (4) and (6) was implemented for testing in 2002 on the HF SNR, but with only manual activation of the real-time search for optimal $\bar{\alpha}, \bar{\beta}$, and $b$. Also, the gains $\mu$ and $\gamma$ were probed and adjusted manually during the tests in 2002. But, for the Self-Tuning version of MSEK, multi-scale based variances and third-moments of pressure oscillations are used as activation functions in the Parameter Tuning block to automatically trigger the searches and fine-tunings as described. Upon search activation, the search diameter is adjusted according to a Time-Scale Averaged Pressure Variance computed with a sufficiently large time-constant for averaging. Also, the gains " $\mu$ " and " $\gamma$ " in the Self-Tuning version are adjusted automatically, and continuously based on a Time-Scale Averaged Pressure Variance computed with a much smaller time-constant for averaging.

\section{Controller Evaluation}

First, the "Basic MSEK" (the core logic of the "Self-Tuning MSEK") was validated and successfully demonstrated at UTRC in 2002 with the HF SNR, also using the high-frequency valve designed by the Georgia Institute of Technology for fuel pulsing (Fig. 5) (Ref. 6). The validation for this part of the controller will be briefly summarized in the next subsection. 


\section{A. Model-Based Validation and Experimental Results of the "Basic MSEK"}

The SNR was operated at a pressure, temperature, and fuel-air ratio corresponding to a mid-power engine condition $\left(\mathrm{T} 3=770{ }^{\circ} \mathrm{F}, \mathrm{P} 3=200\right.$ psia, fuel-air ratio $\left.=0.03\right)$. This HF configuration of the SNR (Fig. 2) exhibited roughly the same engine combustor instability behavior as shown in Figure 3. The SNR combustor pressure was sensed about 2 inches downstream of the fuel injector. The control algorithms were implemented on a real-time processor for an I/O rate of $5000 \mathrm{~Hz}$. As in the LF case, the parameters of the (reduced-order) model for that HF configuration (to be shown in the next paragraph) are with "psi" as unit for the pressure feedback, and Volt for the fuel-modulation command $u_{\mathrm{n}}$ issued to the fuel-valve. This valve command is limited to 2 Volts in amplitude for the HF rig, which corresponds to the maximum valve displacement allowed about the mean positions. The mean valve position was set for the optimal flow number regarding control-authority.

Without loss of generality, the measurement gain and the internal feedback gain for the dominant mode (Eq. (1b) and (1e), respectively) were normalized and balanced such that: $c_{2}=h_{22}=1.0$. With this balance normalization and the mentioned unit choice, the rest of model parameters were then chosen to match empirical open-loop characteristics of the combustor (e.g., Fig. 3). And, this was done in the order of matching mode 2 characteristics and responses to fuel modulations first, as it is more dominant, and then mode 1:

First, $p_{\mathrm{o}}=20.0 \mathrm{psi} ; T=0.0002 \mathrm{sec} ; \kappa_{1}=\kappa_{2}=0.6 p_{\mathrm{o}}=12.0 ; g=0.01 ;$ and, $b=0.2322$. Next, $\omega_{2}=$ $3456 \mathrm{radians} / \mathrm{sec} ; \zeta_{2}=0.04$; and, $p_{\max }^{\prime}=0.09 p_{0}$ (for matching mode 2 characteristics). Then, $\omega_{1}=1822 \mathrm{radians} / \mathrm{sec}$; $\zeta_{1}=0.3 ; c_{1}=0.8 ; h_{11}=1.0$ (for mode 1 characteristics); and $h_{12}=h_{21}=0.1$ (Modal-couplings in the HF configuration of the SNR apparently were low); $\bar{\tau}_{\mathrm{a}}=0.0038 ; \bar{\tau}_{\mathrm{b}}=0.0033 ; \bar{\tau}_{\mathrm{c}}=0.0002$ seconds (Empirical, mean values).

Figure 11 shows the predicted Amplitude Spectra and Probability Density of combustor pressure oscillations; the control-ON compared to the control-OFF case for a rather high level of disturbances comparable to the actual $H F$ SNR environment. Figure 12 shows similar results from test data in 2002 with the Basic MSEK controller on the HF SNR. Both the model prediction and test data show a reduction of about 30 percent in pressure oscillation amplitude at the HF peak. Reference 6 also includes some analytical results showing the controlled "absolute stability" achieved by this core logic. Note that, controlled "absolute stability" is possible if the time-delay of fuel-modulation effect (" $\tau_{b}$ ", Eq. (1a)) are comparable to, if not less than, that of the internal heat-release feedback (" $\tau_{a}$ ").
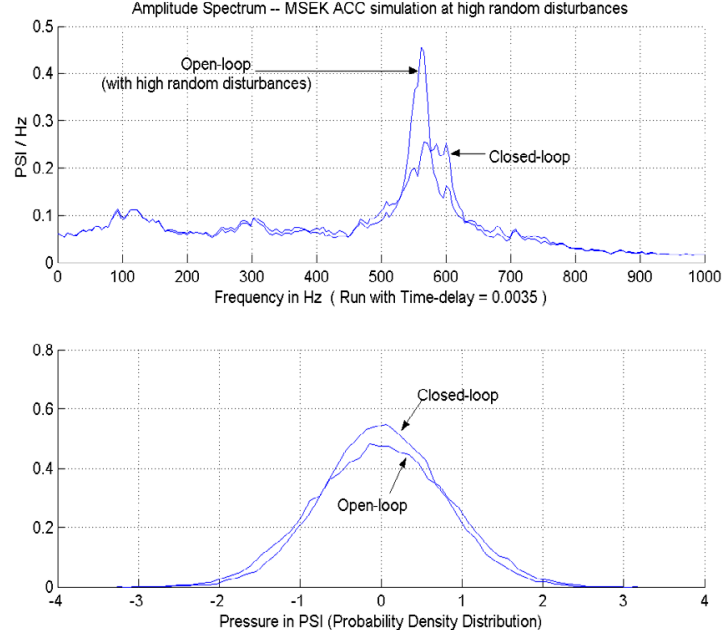

Figure 11.-MSEK, HF SNR Simulation: pressure oscillations amplitude-spectrum (Upper) and probability density of pressure oscillations

(Lower), with/without control.

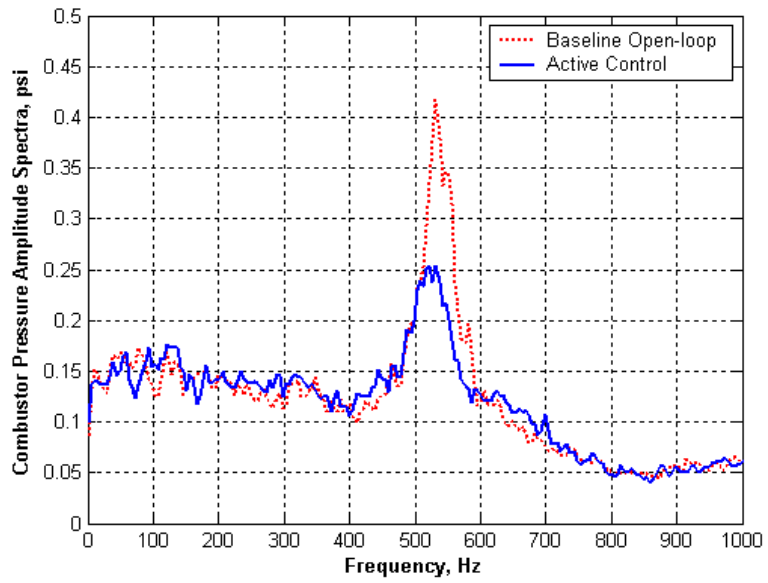

Figure 12.-(9-11-02 HF SNR test) Amplitude spectra showing the effects of the Basic MSEK controller on the combustion instability peak pressure oscillation. 


\section{B. Model-Based Validation and Experimental Results of the "Self-Tuning MSEK"}

The "Mid-Frequency" (MF) and "Low-Frequency" (LF) SNR configuration were also used in the 2004 ACC demonstrations at NASA Glenn. The MSEK and it self-tuning features and parameters were designed and validated on the Reduced-Order model shown in sections II and III, and also on a Sectored 1-D model (1-D CFD, by Paxson, Ref. 14) before testing on the SNR (see Figs. 13 and 14 for some validation results of this controller on the Sectored 1-D model).

The main findings for the optimal operation of the Self-Tuning MSEK on the LF rig include: The setting of the valve "flow-number" for optimal fuel-modulation effects; the nominal search steps (for $\bar{\alpha}, \bar{\beta}$, and $b$ ); the threshold for initiating global search; and lastly the optimal loop-shaping fraction " $l$ ". Initially, "l" had been set at 0.5 . With that value of "l" and the optimal search steps and threshold, the automatic tuning actions of the MSEK controller was able to suppress the instabilities by 80 percent after having been engaged for about $5 \mathrm{sec}$. However, such suppression could not be held steady with setting $l=0.5$ because of some low-frequency oscillations (see Fig. 15). Such low-frequency oscillations are most likely due to the mutual "beating" between mode-1 instability ( $315 \mathrm{~Hz})$ with the fuel-modulation effects by the controller near $320 \mathrm{~Hz}$ that appeared to have suppressed mode-1 almost completely. The left subplot of Figure 15 shows the amplitude-spectrum of the pressure oscillations of the middle subplot for this case; and the right subplot shows the spectrum of the valve command signal. This command spectrum is broadband, but concentrated mostly around $300 \mathrm{~Hz}$. This setting reduced the peak of the pressure amplitude spectrum down to about $1.2 \mathrm{psi} / \mathrm{Hz}$. This is a significant reduction of the instability, as compared to when the controller is turned off, which is about $11 \mathrm{psi} / \mathrm{Hz}$ (e.g., the case of Fig. 6).

When "l" is set near 1.0 such as in the case of Figure 16, the resulting command spectrum was sharper and more concentrated at $300 \mathrm{~Hz}$. But, the instabilities appeared to be suppressed by only 70 percent and yet not steady, again because of some low-frequency oscillations. Although the original instability that had been at $315 \mathrm{~Hz}$ was

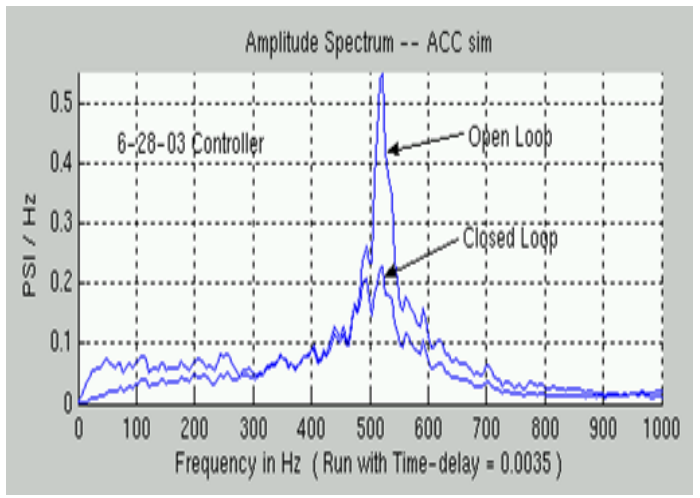

Figure 13.-MSEK on a HF Sectored 1-D model.

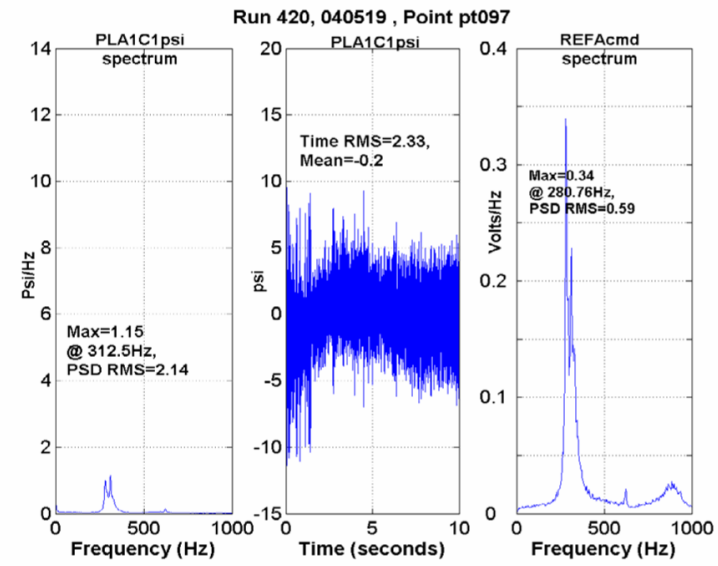

Figure 15.- Preliminary test results of the self-tuning MSEK on the LF (315 Hz) SNR-May 19, 2004.

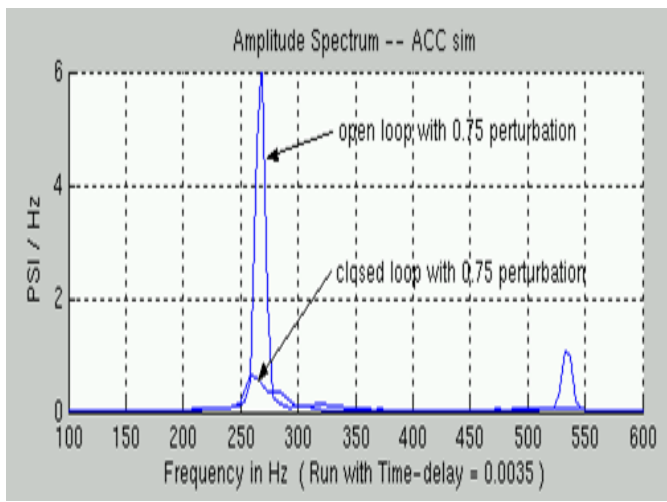

Figure 14.--MSEK on an LF Sectored 1-D model.

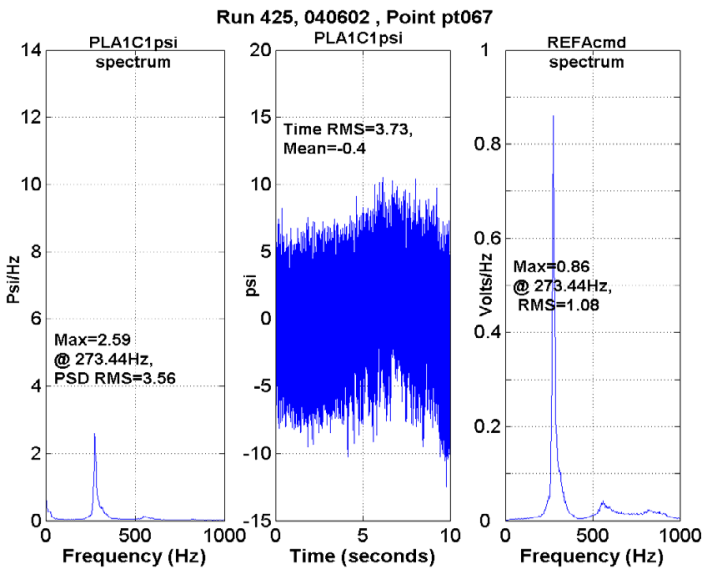

Figure 16.-Preliminary test results of the self-tuning MSEK on the LF (315 Hz) SNR-June 2, 2004. 
eliminated entirely this time, the fuel-modulation produced an undesirable disturbance at $273 \mathrm{~Hz}$ with unsteady amplitude.

The instability suppression by the Self-Tuning MSEK was best and steady when the command was more broadband towards the high-frequency range as shown in Figure 17. This was when "l" was set near 0.1. Under this setting, the controller was able to suppress the oscillation (rms) amplitude from about 10 psi (e.g., shown in the upper subplots of Fig. 6) to about 1.7 psi. This was so because the instabilities were concentrated mostly at $315 \mathrm{~Hz}$ while fuel-modulation with the GT valve was able to affect this frequency most deeply over a fairly wide range of commanded frequencies. Apparently, fuel-modulation at high-frequencies for the LF configuration would not excite the higher modes, but instead affects significant energy transfer towards suppressing the low-frequency instability. So, the role of " $0 \leq l \leq 1$ " is clearly that of typical loop-shaping with a sensitive control parameter. This seems to be essentially the same phenomenon discovered previously by Kopasakis on the same rig when focusing control on the "2nd harmonic" with an adaptive phase-shifting controller (Ref. 3).

Lastly, the MF SNR exhibited an instability at $340 \mathrm{~Hz}$ (Fig. 18(a)), a frequency fairly close to the LF; but, its nature is more like that of the HF instability. The plots shown in Figure 18(b) are from a preliminary study of the MSEK on the MF configuration that show some moderate reduction in instabilities; about 50 percent reduction of the amplitude spectra peak relative to the noise floor compared to open-loop. Also, the rms amplitude of overall pressure oscillation was reduced to about 1.4 psi. This is probably the limit for active fuel-modulation effect with this valve (Fig. 5) for this case. This is consistent with the 2002 results on the $H F$ rig, and the test results on the $L F$ rig shown in Figure 17 (Noting that, the time-plot in Fig. 17 also showed peaks near 4 psi, comparable to the time-plot of Fig. 18(b)).

\section{MSEK Model-Based Control Dynamics Discussion \\ The LF Configuration experimental result of} Figure 17 is an important case that had not previously been simulated. It was found from simulating this case that the non-linear heat-release over the frequency range between mode- 1 and mode-2 seems to be crucial for the steady suppression of the instability. Therefore, some numerical results of the MSEK on the validated

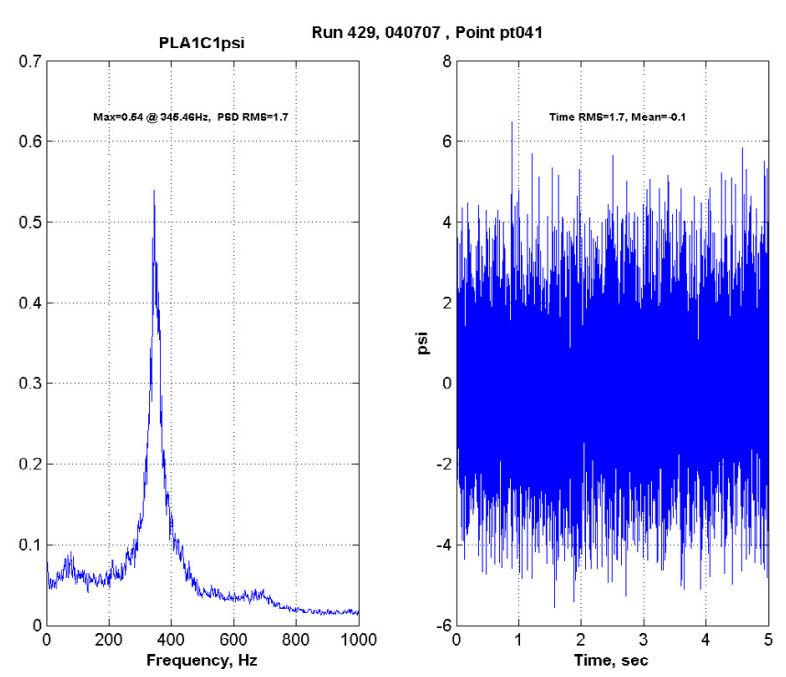

Figure 18(a).-MF self-sustained instability.

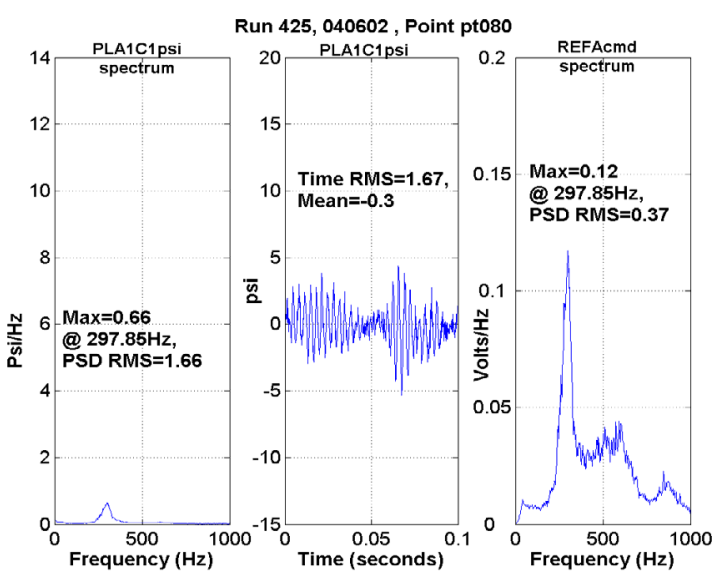

Figure 17. - The LF $(315 \mathrm{~Hz})$ suppressed by the self-

Tuning MSEK controller-June 2, 2004 Test.

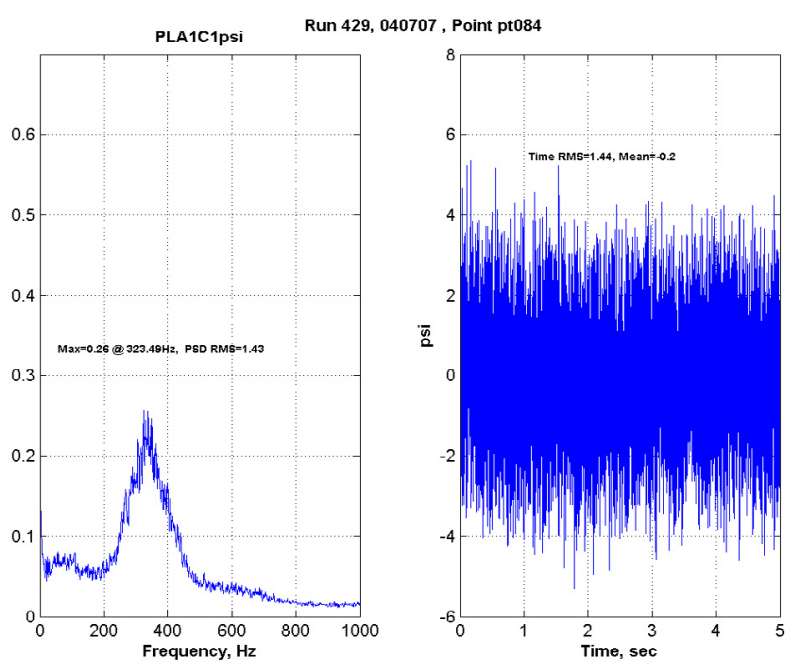

Figure 18(b).-MF instability suppressed by MSEK. 
reduced-order model are presented here for further understanding of the control dynamics and for validating the model against test data for the close-loop case. First, Figure 19(a) shows the self-tuning control in action on the LF model, and the combustion pressure being quickly reduced to a level comparable to an actual test case on the LF SNR (Compare Fig. 17 of an actual test case with a "zoomed-in" portion of the simulation run case in Fig. 19(a), as shown in Fig. 19(b)). The "mode-1 fraction, l" of Equations (4) and (6) of the Damper and Tone Suppressor actions, used as a "loop-shaping" parameter, is shown in the third subplot of Figure "19(a)". Note that, comparing to those results within the first second time interval when the control had not been activated, the pressure oscillations afterwards have never been worsened by the tuning actions. Figure 19(c) shows the difference in the unsteadiness of heat-addition of two zoomed-in time portions in Figure 19(a): one portion at $0.5 \mathrm{sec}$ from start when the controller had not been activated; and another at time $20 \mathrm{sec}$ when the controller parameters has already converged to a good region. It shows that, fuel-modulation under good control likely has the effects of preventing the heat addition from dropping too low in resonance with the acoustics. Thus, fuelmodulation might also be effective for stabilizing the flame when the combustor is operated near its "leanblowout" margin. Such potential application of active fuel-modulation (e.g., to "lean-direct injection combustors), however, needs further investigations.

\section{Conclusion}

This combustion stability control scheme, the selftuning Multi-Scale Extended Kalman (MSEK) controller, has been used to experimentally suppress thermo-acoustic instabilities up to 90 percent. The controller employed a Quadratures Filter Bank to perform multi-scale analyses in real-time on the sensed pressure signals for control feedback. An approximate evolution equation of the extracted "Quadratures" (representing the acoustics states) was used to formulate a Kalman predictor for these acoustics states for the damper logic. The variable gains, phase bias, and other automatic control tuning logics were based on such multi-scale analysis. This model-based, self-tuning control scheme was thoroughly tested on a liquid-fuel combustor rig operated at engine-like conditions, for three configurations emulating different cases of combustion instability. The combustion control study on this rig confirmed earlier NASA observations and provided a plausible explanation about an important internal mechanism of cross-frequency couplings in combustion thermo-acoustics. The dynamic couplings are very prominent in its Low-Frequency configuration, and in general could be strongly affected by local fuel/air modulations. The reduced-order thermo-acoustics model for control development, using a simplistic parametric
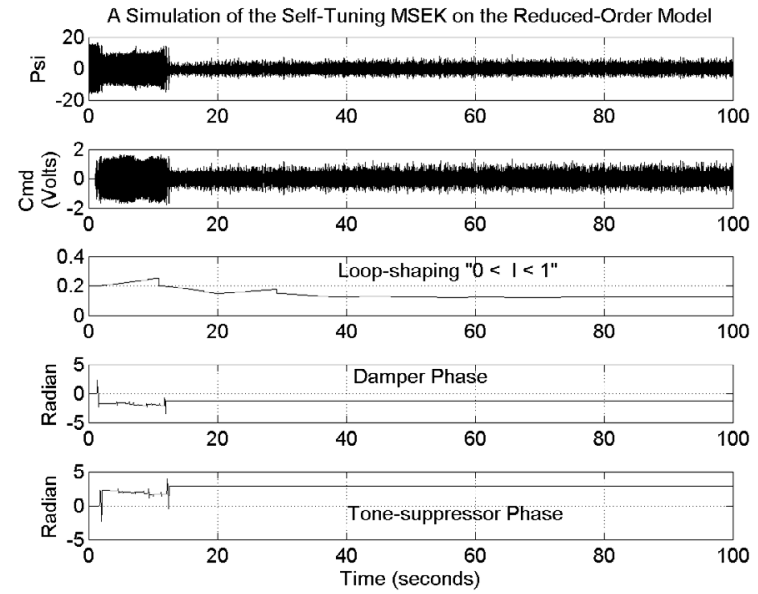

Figure 19(a).- -Simulation results with the selftuning MSEK controller on the reduced-order plant

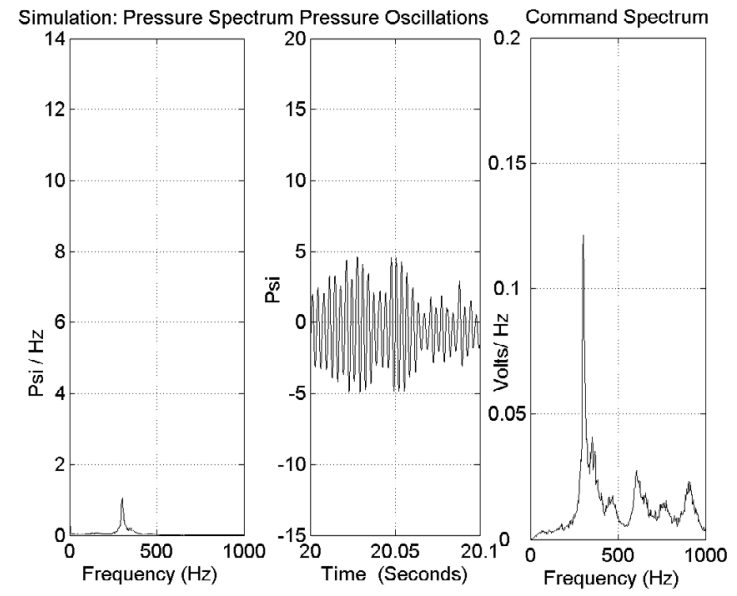

Figure 19(b).- - Simulation analysis of the selftuning MSEK controller (see Fig 19(a)).
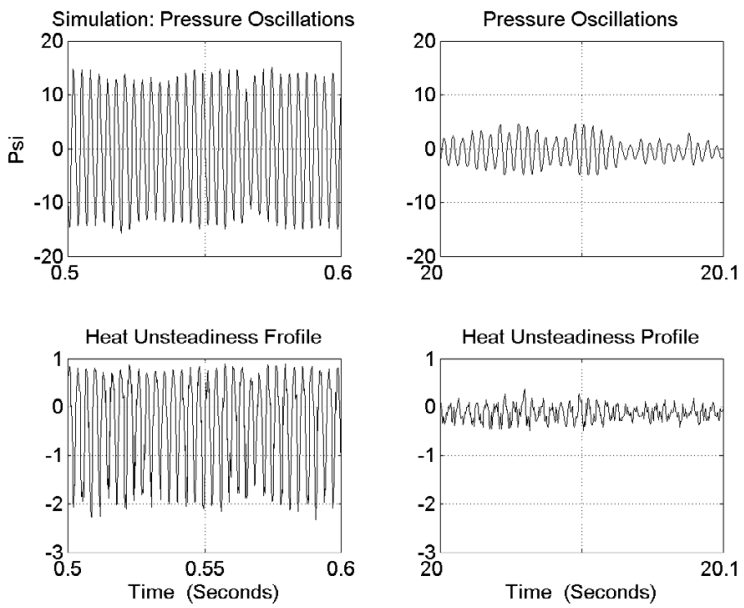

Figure 19(c).- - Instability and heat unsteadinesssimulation (see Fig 19(a)). 
formulation for the non-linear heat-fluctuations that drive the acoustics, exhibits the same response characteristics of the rig on both the frequency-domain and time-domain. The self-tuning MSEK based on this model was able to adjust it own parameters and control gains to suppress nearly completely the combustion instabilities by modulating the fuel injector flow with a high-frequency valve. The main contributors to the effective suppression of combustion instabilities as demonstrated by this controller were the control-authority of the high-bandwidth fuel valve and the cross-frequency couplings in heat-release under fuel modulation.

\section{References}

1. Lefebvre, A.H.: Gas Turbine Combustion, 2nd edition, Taylor \& Francis, 1999.

2. Annaswamy, A.M. and Ghoniem, A.F.: "Active Combustion Instability: Theory and Practice," IEEE Control Systems Magazine, December 2002, Vol. 22, Number 6.

3. Kopasakis, G., DeLaat J.C., and Chang C.T.: "Validation of an Adaptive Combustion Instability Control Method for Gas-Turbine Engines," presented at the AIAA/ASME/SAE/ASEE Joint Propulsion Conference, Fort Lauderdale, Florida, July 2004, NASA/TM-2004-213198, AIAA-2004-4028.

4. Kopasakis, G.: "Systems Characterization of Combustor instabilities with Controls Design Emphasis," presented at the 42nd AIAA Aerospace Sciences Meeting and Exhibit, Reno, Nevada, January 2004, NASA/TM-2004212912, AIAA-2004-0638.

5. Kopasakis, G.: "High Frequency Adaptive Instability Suppression Controls in a Liquid-Fueled Combustor," AIAA 39th Joint Propulsion Conference and Exhibit, Huntsville, AL, July 2003, NASA/TM-2003-212535, AIAA-2003-9581.

6. Le, D.K., Delaat J.C., and Chang C.T.: "Control of Thermo-Acoustics Instabilities: The Multi-Scale Extended Kalman Approach," NASA/TM-2003-212536, AIAA-3003-4934 presented at the 39th Joint Propulsion Conference, Huntsville, Alabama, July 20-23, 2003.

7. Meyer Y., 1991, Wavelets: Algorithms and Applications, English translation and revision by Robert D. Ryan, SIAM edition 1994.

8. Houdre, C., 1993: "Wavelets, probability, and statistics: Some bridges," Wavelets Mathematics and Applications (Studies in Advanced Mathematics), edited by J.J. Benedetto and Michael W. Frazier, GRC Press, 1994 edition.

9. Misawa, E.A. and Hedrick, J.K.: "Nonlinear Observers-A State-of-the-Art Survey," Transaction of the ASME, Journal of Dynamic Systems, Measurement, and Control, Vol. 111, pp. 344-352, September 1989.

10. Jazwinski A.H., "Stochastic Processes and Filtering Theory," Mathematics in Science and Engineering Vol. 64, Academic Press, 1970.

11. Cohen, J.M. et al.: "Experimental Replication of an Aeroengine Combustion Instability," International Gas Turbine and Aero-Engine Congress and Exhibition, Munich, Germany, 2000.

12. DeLaat, J.C. and Chang, C.T.: "Active Control of High Frequency Combustion Instability in Aircraft GasTurbine Engines," presented at the 16th International Symposium on Air breathing Engines, Cleveland, Ohio, August 31-September 5, 2003.

13. Barooah, P., Anderson, T.J., and Cohen, J.M.: "Active Combustion Instability Control with Spinning Valve Actuator," Proceedings of ASME Turbo Expo, Amsterdam, Netherlands, 2002. GT-2002-30042.

14. Paxson, D.E.: "A Sectored-One-Dimensional Model for Simulating Combustion Instabilities in Premix Combustors," presented at the 38th AIAA Aerospace Sciences Meeting \& Exhibit, Reno, Nevada, January 2000, NASA/TM-1999-209771, AIAA-2000-0313. 


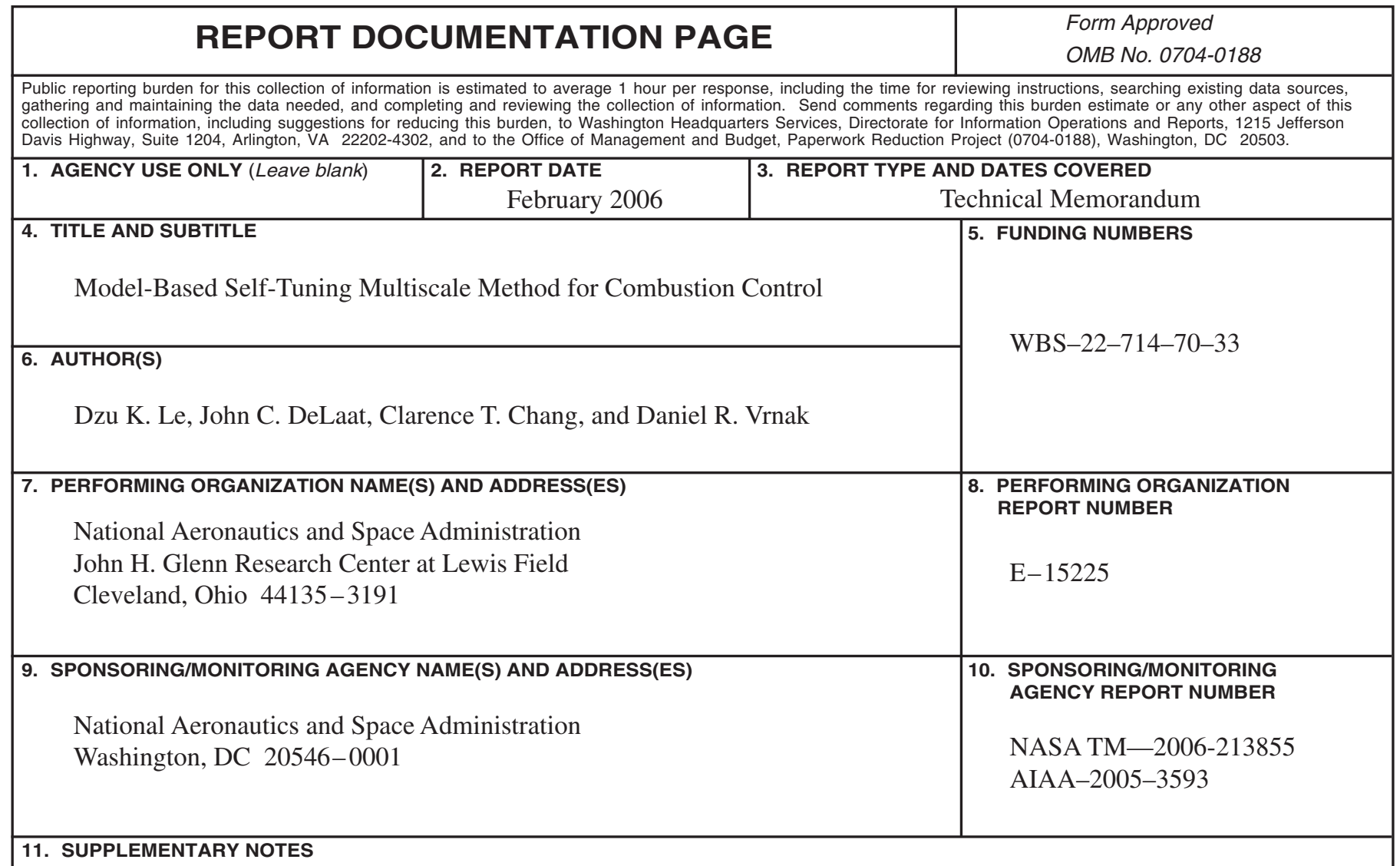

Prepared for the 41st Joint Propulsion Conference and Exhibit cosponsored by the AIAA, ASME, SAE, and ASEE, Tucson, Arizona, July 10-13, 2005. Responsible person, Dzu K. Le, organization code DEI, 216-433-5640.

\begin{tabular}{l|l}
\hline 12a. DISTRIBUTION/AVAILABILITY STATEMENT & 12b. DISTRIBUTION CODE \\
Unclassified - Unlimited & \\
Subject Category: 07 & \\
Available electronically at http://gltrs.grc.nasa.gov & \\
This publication is available from the NASA Center for AeroSpace Information, 301-621-0390. &
\end{tabular}

\section{ABSTRACT (Maximum 200 words)}

A multi-scale representation of the combustor dynamics was used to create a self-tuning, scalable controller to suppress multiple instability modes in a liquid-fueled aero engine-derived combustor operating at engine-like conditions. Its selftuning features designed to handle the uncertainties in the combustor dynamics and time-delays are essential for control performance and robustness. The controller was implemented to modulate a high-frequency fuel valve with feedback from dynamic pressure sensors. This scalable algorithm suppressed pressure oscillations of different instability modes by as much as 90 percent without the "peak-splitting" effect. The self-tuning logic guided the adjustment of controller parameters and converged quickly toward phase-lock for optimal suppression of the instabilities. The forced-response characteristics of the control model compare well with those of the test rig on both the frequency-domain and the timedomain.

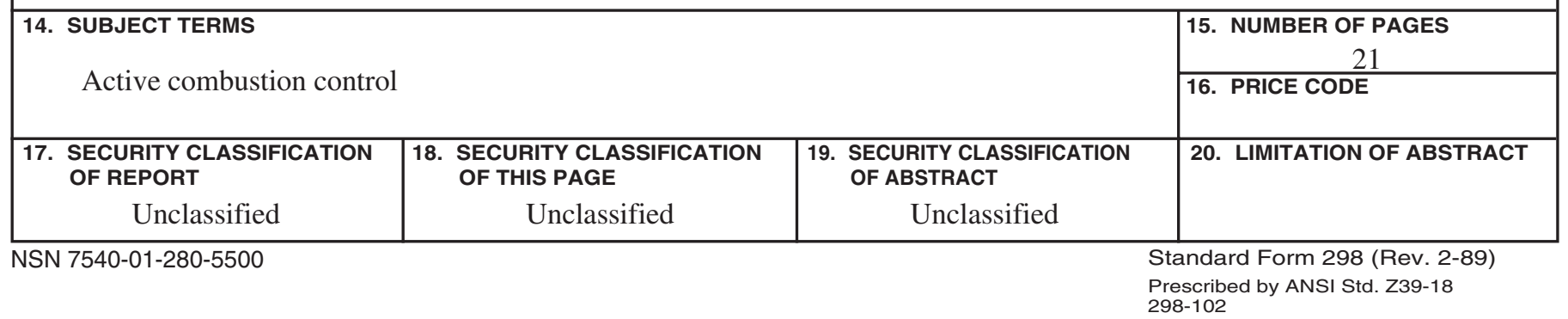



